\title{
Estimación de la Masa Muscular de los Miembros Apendiculares, a Partir de Densitometría Fotónica Dual (DEXA)
}

\author{
Muscle Mass Estimation of Appendicular Limb, for Dual Energy X Ray Absorciometry (DEXA)
}

\author{
"Fernando Javier Rodríguez Rodríguez; ${ }^{* *}$ Atilio Aldo Almagià Flores \& ****Francisco José Berral de la Rosa
}

\begin{abstract}
RODRÍGUEZ, R. F. J.; ALMAGIÀ, F. A. A. \& BERRAL, R. F. J. Estimación de la masa muscular de los miembros apendiculares, a partir de densitometría fotónica dual (DEXA). Int. J. Morphol., 28(4):1205-1210, 2010.

RESUMEN: El objetivo de esta investigación es crear una fórmula de regresión que permita establecer la cantidad de masa muscular por región considerando como "gold estándar" al método DEXA. Se evaluaron 20 ciclistas entrenados de nivel amateur considerando 45 variables antropométricas, para posteriormente aplicar el método DEXA. A través de ecuaciones de regresión en la plataforma SPSS 17, se estableció una fórmula de estimación para masa muscular de los miembros superiores donde, MMES $(\mathrm{kg})=(($ Talla - Pliegue Triceps $) \mathrm{x}\left(0,043 \mathrm{xPerímetro} \mathrm{Brazo} \mathrm{relajado}{ }^{2}\right)-$ Peso) / 1000, con un valor $\mathrm{r}=0,83$ y un error estimado de $0,61 \mathrm{~kg}$. Para la masa muscular de

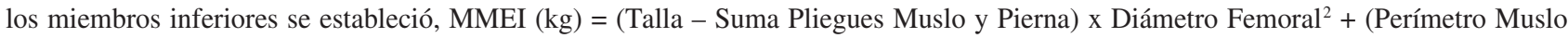
Medio + Perímetro Pierna) / 1000, con un r= 0,74 y un error de estimación de 1,2 kg. La determinación de estos componentes por segmento, mejoran la especificidad del conocimiento anatómico de individuos de distintas poblaciones, especialmente en deportistas, quienes difieren su distribución de tejidos de acuerdo a la disciplina deportiva y a muy bajo costo, como lo hace la antropometría.
\end{abstract}

PALABRAS CLAVE: Antropometría; Masa muscular; Densitometría Fotónica Dual.

\section{INTRODUCCIÓN}

Las características físicas están ligadas al rendimiento deportivo, debido a la asociación que se puede establecer entre la distribución de los componentes morfoestructurales (grasa, músculo, hueso, etc), y el desempeño motor, considerando, por ejemplo la eficiencia mecánica que ofrece un menor peso en grasa y más fuerza con más músculo, siendo fundamental el conocimiento de estos componentes corporales para su posterior cuantificación.

En los años noventa comienzan a realizarse estudios para el desarrollo de un método más moderno y no invasivo que permite obtener de manera precisa la cantidad de masa ósea, masa grasa y masa magra (Aloia et al., 1995) a través del método DEXA, Dual Energy X-ray Absorciometry (Haarbo et al., 1991; Jensen et al., 1993; Brunton et al., 1993; Kohrt et al., 1998).
Kim et al., fueron capaces de estimar la masa muscular total por medio del método DEXA (Kim et al., 2002, 2004; Shih et al., 2000; Levine et al., 2000; Visser et al., 1999), pero no consiguieron dividir este compartimiento por segmentos corporales. Por este motivo, el desarrollo del método antropométrico de fraccionamiento por regiones y validado por DEXA, permitiría realizar comparaciones específicas entre la manera en que se distribuyen los tejidos (Lohman \& Chen, 2005), y su influencia en el rendimiento físico, como la masas grasa y muscular, de acuerdo a la actividad deportiva que realiza cada individuo, teniendo de este modo información de la distribución y desarrollo físico diferenciado, que el simple análisis de la totalidad de cada componente de la composición corporal, no es capaz de proporcionar. Esto permitirá ampliar el conocimiento morfoestructural y su aplicación a cualquier tipo de población.

\footnotetext{
* Escuela de Educación Física, Facultad de Filosofía y Educación, Laboratorio Motricidad Humana. Laboratorio de Antropología Física y Anatomía Humana, Instituto de Biología, Facultad de Ciencias, Pontificia Universidad Católica de Valparaíso, Chile.

** Laboratorio de Antropología Física y Anatomía Humana, Instituto de Biología, Facultad de Ciencias, Pontificia Universidad Católica de Valparaíso, Chile.

**** Departamento de Deporte e Informática, Facultad del Deporte, Universidad Pablo de Olavide, España. Colaborador Laboratorio de Antropología Física y Anatomía Humana, Instituto de Biología, Facultad de Ciencias, Pontificia Universidad Católica de Valparaíso, Chile.
} 


\section{MATERIAL Y MÉTODO}

Fueron evaluados 20 ciclistas recreativos, entre los 20 y 33 años de edad y una media de $25,8 \pm 4,2$ años, todos voluntarios bajo consentimiento informado, quienes accedieron al estudio como una muestra no aleatoria e intencionada. Los criterios de inclusión fueron tener una buena salud, y entrenar al menos tres veces por semana, no padecer factores de riesgo cardiovascular y estar en categoría de IMC normal, lo cual se determinó previo a la evaluación. Todos los sujetos eran residentes de la V Región de Valparaíso de Chile.

Las variables antropométricas escogidas, correspondieron al protocolo de evaluación completa de ISAK, que incluye variables básicas, perímetros musculares apendiculares y axiales, diámetros óseos y pliegues cutáneos. Cada una de estas variables se utilizaron para estimar las distintas masas según los autores escogidos para este estudio. La evaluación antropométrica se realizó en las primeras horas de la mañana, con la menor ropa posible y después del vaciado urinario, bajo el protocolo de marcaje de la International Society for the Avancement of Kineatropometry (ISAK) y de evaluación descritos por Donald Drinkwater (Drinkwater, 1984) y William Ross (Ross et al., 1982) para el protocolo de completo de variables.

Se analizaron las variables antropométricas, donde las estadísticas calculadas fueron, promedio, desviación estándar, mediana, mínimo, máximo y percentiles 5\%, 15\%, $25 \%, 50 \%, 75 \%, 85 \%$ y $95 \%$, siendo éstas, en antropometría, las medidas más utilizadas para propósitos de ubicación o clasificación de los individuos cuando atienden a características tales como peso, estatura, etc.

La evaluación DEXA (Dual Energy X-ray Absorsiometry) que significa en español absorciometría de rayos $\mathrm{X}$ de doble frecuencia funciona enviando un haz delgado e invisible a dos intensidades distintas, los cuales atraviesan los tejidos de distintas densidades o captar solo el tejido óseo y el resto del tejido correspondiente a masa libre de grasa.

Esta evaluación se realizó con el densitómetro LUNAR DPX-MD de Lunar Radiation Corporation, Madison, WI, USA. Esta posee una colimación de 1,68 mm y a una intensidad de $150 \mu \mathrm{A}$. Utiliza rayos X con un haz de lápiz DXA y con una fuente de potencial constante de $76 \mathrm{kV}$.

\section{RESULTADOS}

A través del método DEXA, evaluado en los sujetos del estudio, fue posible determinar la masa adiposa (MA), la masa libre de grasa (MLG) y la masa ósea seca (MOS), esta última no representa la masa ósea MO total, por lo tanto fue necesario estimar la masa ósea húmeda (MOH), la cual es la que estima la antropometría.

\begin{tabular}{|c|c|c|c|c|c|}
\hline & Masas segmentadas & Prome dio & DS & Máximo & Mínimo \\
\hline \multirow{5}{*}{ 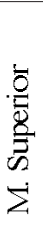 } & Masa adiposa & 1,5 & 0,7 & 3,5 & 0,4 \\
\hline & Masa libre de grasa & 7,4 & 1,1 & 9,6 & 5,7 \\
\hline & Masa ósea seca & 0,5 & 0,1 & 0,6 & 0,3 \\
\hline & Masa ósea húmeda & 0,8 & 0,1 & 1,0 & 0,6 \\
\hline & Masa muscular & 6,6 & 1,1 & 8,6 & 5,0 \\
\hline \multirow{5}{*}{ 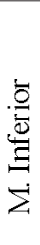 } & Masa adiposa & 3,5 & 1,1 & 5,6 & 1,7 \\
\hline & Masa libre de grasa & 19,6 & 2 & 23,3 & 16,2 \\
\hline & Masa ósea seca & 1,3 & 0,2 & 1,6 & 0,9 \\
\hline & Masa ósea húmeda & 2,4 & 0,3 & 2,9 & 1,7 \\
\hline & Masa muscular & 17,2 & 1,8 & 21,1 & 14,1 \\
\hline \multirow{6}{*}{$\begin{array}{l}\stackrel{0}{0} \\
\stackrel{\Xi}{E}\end{array}$} & Masa adiposa & 6,1 & 2,7 & 12,0 & 2,5 \\
\hline & Masa libre de grasa & 28,1 & 2,6 & 33,4 & 24,2 \\
\hline & Masa ósea seca & 1,2 & 0,2 & 1,6 & 0,9 \\
\hline & Masa ósea húmeda & 4 & 0,7 & 5,2 & 3,1 \\
\hline & Suma MLG AP & 27 & 2,8 & 31,7 & 22,3 \\
\hline & Masa muscular total & 31,5 & 3,1 & 36,7 & 26,4 \\
\hline
\end{tabular}

Tabla I. Variables determinadas por DEXA en miembros superiores, miembros inferiores y tronco, y sus resultados estadísticos. Promedio; Desvío Estandar (DS); Valor Máximo (Máximo) y Valor Mínimo (Mínimo). La masa muscular total es estimada a partir de la formula de Heymsfield et al. (1990). 
A partir de la determinación de la MOS, Steven Heymsfield, propone que ésta, representa el 55\% del peso total del hueso, por tanto para obtener la $\mathrm{MOH}$ se debe dividir la MOS por 0,55 o multiplicar por 1,82 .

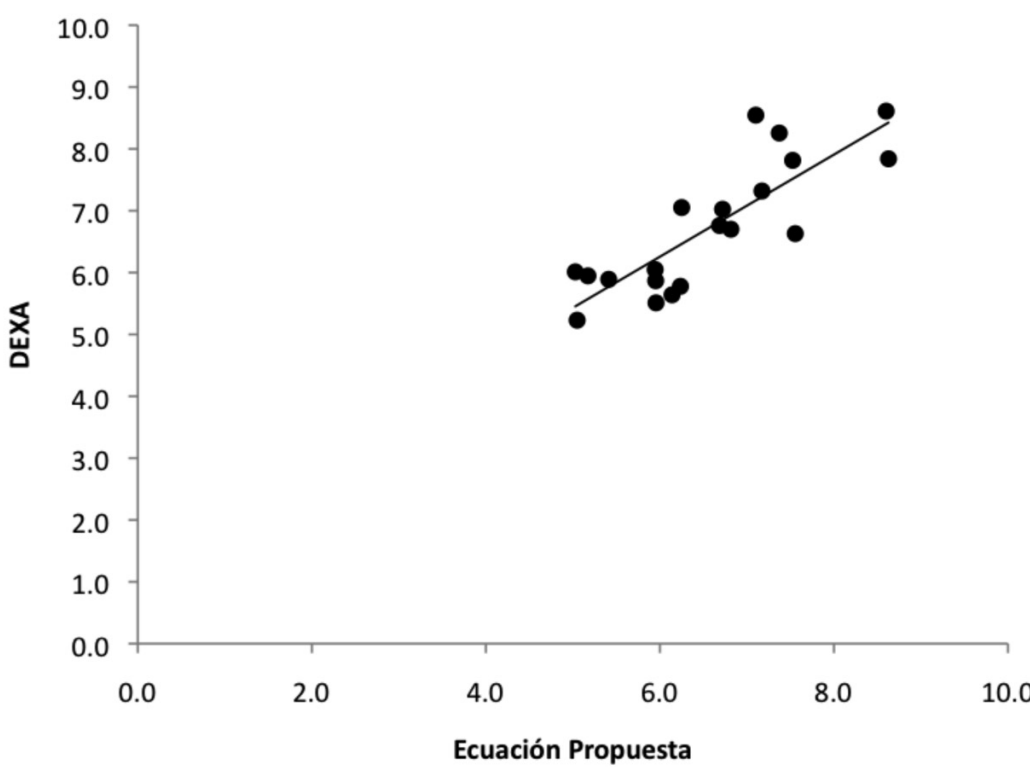

Fig. 1. Correlación entre los valores de MMES estimado por ecuación de regresión y determinado por DEXA. $r=0,83$.

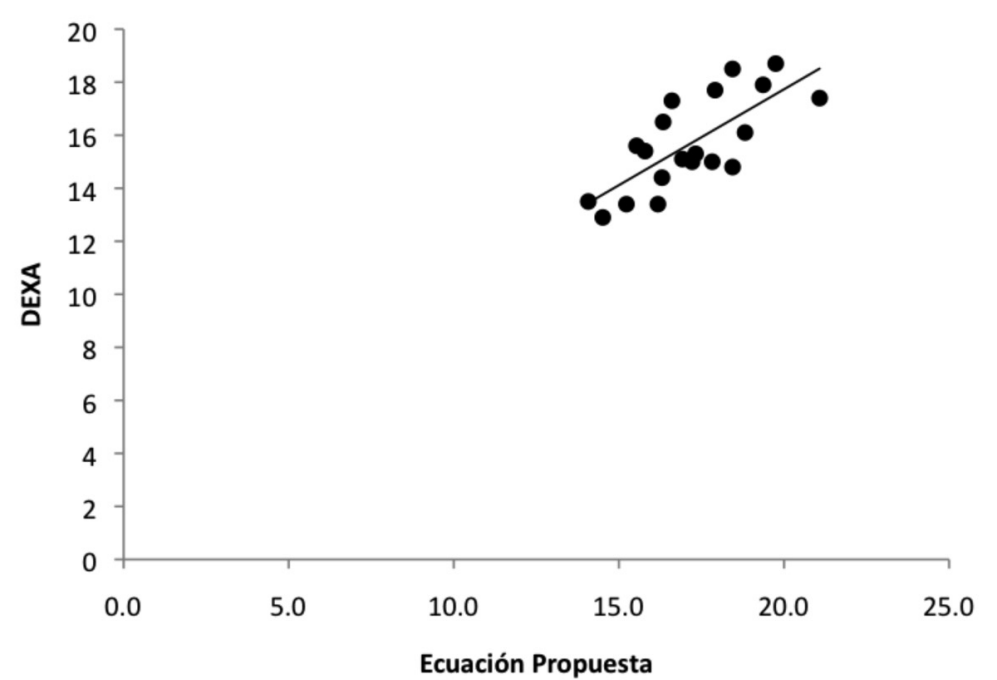

Fig. 2. Correlación entre los valores de MMEI estimado por ecuación de regresión y determinado por DEXA. $r=0,74$.

La masa corporal de los miembros del cuerpo humano está compuesta por masa ósea (MO), masa muscular (MM), masa adiposa (MA) y masa de piel (MP) que representa aproximadamente el 5\% del peso total del cuerpo (Kerr, 1988).
Obtenidos los componentes precisos de MA y MLG por DEXA, se determinó cuanto de la MLG corresponde a MO y cuanto a MM. Para esto se restó la MOH de la MLG y se obtuvo el contenido de MM del miembro.

Es decir, Masa total= MA + MLG, donde, $\mathrm{MLG}=\mathrm{MO}+\mathrm{MM}$ y la $\mathrm{MM}=\mathrm{MLG}$ $-\mathrm{MOH}$.

De acuerdo a esto se establecieron los valores de MM de los miembros superiores e inferiores (Tabla I). La masa muscular total de los sujetos, fue obtenida a partir de la suma de la MLG de solo los miembros superiores e inferiores medidas por el método DEXA (Tabla I), a través de la fórmula desarrollada por Kim et al. (2004), donde se obtiene un $\mathrm{r}=0,96$ entre la masa muscular obtenida por la fórmula de estimación matemática y la determinada por resonancia nuclear magnética (RNM), donde:

Masa Muscular Total $($ MMT $)=(1,19$ - MLG) - 1,01. (Kim, 2002)

Para obtener los valores de MM de los miembros superiores e inferiores en los sujetos del este estudio, se determinó que variables se correlacionan mejor con los valores de MM de DEXA como referencia. Una vez identificadas estas variables con alta correlación, se aplicaron ecuaciones de regresión lineal para determinar que constantes se utilizarán para estimación de la MM por ecuaciones.

De esta manera se obtuvo la siguiente fórmula para la estimación de los kilogramos $(\mathrm{kg})$ de masa muscular de los miembros superiores (MMES) con un $\mathrm{r}=0,83$ (Tabla II).

$$
\begin{gathered}
\text { MMES }=\left(\left((T-P L T) \times\left(0,043 \times(P B R)^{2}\right)\right)-\right. \\
\text { P) } / 1000
\end{gathered}
$$

Donde, $\mathrm{T}=$ Talla; PLT $=$ Pliegue tricipital; PBR = Perímetro brazo relajado; $\mathrm{P}=$ Peso corporal.

Para la estimación de masa muscular de los miembros inferiores (MMEI), se 
RODRíGUEZ, R. F. J.; ALMAGIÀ, F. A. A. \& BERRAL, R. F. J. Estimación de la masa muscular de los miembros apendiculares, a partir de densitometría fotónica dual (DEXA). Int. J. Morphol., 28(4):1205-1210, 2010.

Tabla II. Relación entre masa muscular superior por DEXA y ecuación propuesta.

\begin{tabular}{lcccc} 
Modelo & $\mathrm{R}$ & $\mathrm{R}^{2}$ & Adjuste de $\mathrm{R}^{2}$ & Error Estandar $(\mathrm{kg})$ \\
\hline E. Superior & 0,83 & 0,69 & 0,67 & 0,61
\end{tabular}

Correlación (R); Correlación cuadrada (R2); Ajuste y Error Estandar de Estimación en kg.

Tabla III. Relación entre masa muscular inferior por DEXA y ecuación propuesta.

\begin{tabular}{lcccc} 
Modelo & $\mathrm{R}$ & $\mathrm{R}^{2}$ & Adjuste de $\mathrm{R}^{2}$ & Error Estandar $(\mathrm{kg})$ \\
\hline E. Inferior & 0,74 & 0,54 & 0,51 & 1,2 \\
Correlación (R) ; Correlación cuadrada (R2); Ajuste y Error Estandar de Estimación en kg &
\end{tabular}

obtiene la siguiente formula de regresión en $\mathrm{kg}$ de MM con un $\mathrm{r}=0,74$ (Tabla III).

$$
\text { MMEI }=\left(\left((T-S P M P) x(D F)^{2}\right)+(P M M+P P)\right) / 1000
$$

Donde, $\mathrm{T}=$ Talla; $\mathrm{SPMP}=$ Sumatoria de pliegues del muslo medio y pierna; $\mathrm{DF}=$ Diámetro femoral $; \mathrm{PMM}=\mathrm{Pe}-$ rímetro muslo medio; $\mathrm{PP}=$ Perímetro de la pierna.

\section{DISCUSIÓN}

Se han presentado variados métodos de estimación de la masa muscular (Drinkwater et al., 1985; Kerr; Martin et al., 1990), entre otros, que utilizan como variables esenciales, los perímetros y contornos de los miembros, calculando el valor total de la masa muscular. Además se utilizan perímetros del tronco, como el perímetro del tórax o perímetro abdominal para estimar preferentemente la masa residual.

Por lo tanto, es coherente estimar la masa muscular de los segmentos apendiculares a partir de los perímetros de estos segmentos, como los perímetros del brazo, antebrazo, muslo y pierna.

De esta manera es posible clarificar como se distribuye este componente fundamental para el desarrollo de la actividad física y que cambia según la disciplina deportiva que se realiza.

En este grupo de estudio, se obtiene que la MMSI, representa más de la mitad del peso muscular total (PMT) y que la MMES representa aproximadamente 1/5 del PMT (Fig. 3).

La masa muscular estimada por fórmulas de regresión favorece el conocimiento específico del componente muscular, y de comparar como en el rugby y el futbol, don- de podría haber diferencias importantes en la masa muscular de los miembros superiores.

También permite establecer diferencias con el mismo deportista entrenado o no entrenado, lesionado o en condiciones normales de rendimiento. Una consideración importante se establece al comparar los resultados obtenidos por DEXA y por otros métodos de estimación, donde se aprecia con cual se correlaciona mejor y cuales métodos sobreestiman la masa muscular. Sabiendo que las ecuaciones formuladas se realizan a partir de los resultados de DEXA, es posible establecer que el método desarrollado por Drinkwater presenta similitudes en la estimación con la ecuación planteada en este estudio (Tabla IV).

Las buenas correlaciones obtenidas por ambas ecuaciones, presumen que los valores que se estiman tienen un error bajo que es $0,6 \mathrm{~kg}$ para miembros superiores y 1,2 $\mathrm{kg}$ para miembros inferiores, pero qua al observar los porcentajes de erro, se aprecia un error mayor (Tabla V).

La determinación de estos componentes por segmento, como lo hizo Drinkwater y como son capaces de estimar otros métodos modernos no antropométricos, mejoran la especificidad del conocimiento anatómico de individuos de distintas poblaciones, especialmente en deportistas, quienes difieren su distribución de tejidos de acuerdo a la disciplina deportiva.

Pero aún así, estos métodos siguen siendo de alto costo y de difícil accesibilidad, en comparación a lo económico, fácil y transportable que resulta la técnica antropométrica. Por lo tanto las fórmulas antropométricas que se validan con estos nuevos métodos, abren oportunidades de desarrollar mayor cantidad de conocimientos a partir de estas medidas, que en este estudio aportan a la estimación de la masa muscular, uno de los tejidos más importantes para los deportistas y que se deben seguir desarrollando para la predicción de otros componentes anatómicos o mayor cantidad de segmentos. 


\begin{tabular}{llc}
\hline \multirow{2}{*}{ Métodos } & \multicolumn{2}{c}{ Masa muscular } \\
\cline { 2 - 3 } DEXA & $\mathrm{kg}$ & $\%$ \\
Heymsfield (1982) & 31,5 & 42,5 \\
Drinkwater (1985) & 34,2 & 43,7 \\
Kerr (1988) & $37,6^{*}$ & 45,9 \\
Martin (1990) & $39,0 *$ & $50,6^{*}$ \\
Doupe-Martin (1997) & $44,3 *$ & $52,7 *$ \\
\hline
\end{tabular}

Tabla IV. Resultados de la masa muscular del grupo evaluado a través de diversos métodos de estimación en compararción con DEXA. (*) Significativamente distinto de DEXA con un valor de $\mathrm{p}<0,05$ en una prueba $\mathrm{T}$.

Tabla V. Correlaciones y errores estándar de las ecuaciones para miembros superiores e inferiores en comparación al método DEXA.

\begin{tabular}{ccccc} 
Modelo & $\mathrm{R}$ & $\mathrm{R}^{2}$ & Error Estandar $(\mathrm{kg})$ & \% de Error \\
\hline MMES & 0,83 & 0,69 & 0,61 & $9 \%$ \\
\hline MMEI & 0,74 & 0,54 & 1,2 & $7 \%$
\end{tabular}

\section{DISTRIBUCIÓN DE LA MASA MUSCULAR}

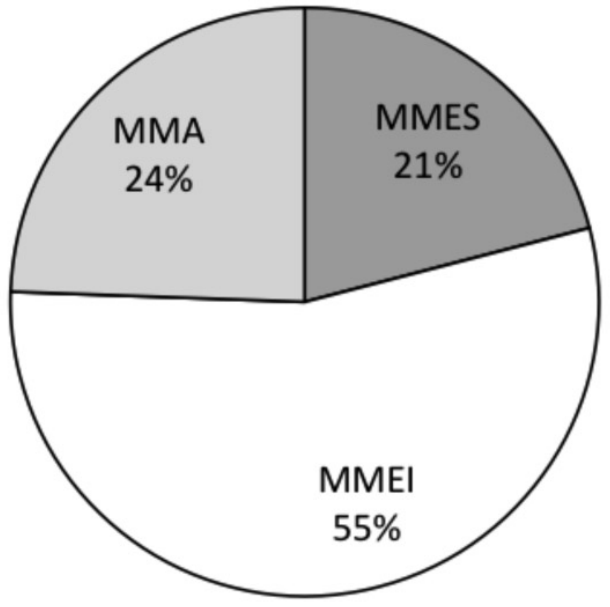

Fig. 3. Distribución de la masa muscular promedio de los sujetos en estudio. MMEI: Masa Muscular de los miembros inferiores; MMES: Masa Muscular de miembros superiores; MMA: Masa Muscular Axial

\section{AGRADECIMIENTOS}

A cada uno de los participantes evaluados en la investigación, a la Escuela de Educación Física por su constante apoyo, al Laboratorio de Antropología Física y Anatomía Humana y a la Dirección Investigación e Innovación de la Pontificia Universidad Católica de Valparaíso.
RODRÍGUEZ, R. F. J.; ALMAGIÀ, F. A. A. \& BERRAL, R. F. J. Muscle mass estimation of appendicular limb, for dual energy X ray absorciometry (DEXA). Int. J. Morphol., 28(4):1205-1210, 2010.

SUMMARY: The aim of this investigation is to create a regression formula that allows establishING the quantity of muscle mass for limb considering "gold standard " to the DEXA method. 20 amateur cyclists trained are evaluated considering 45 anthropometrics measures, later applying the method DEXA across equations of regression in the platform SPSS 17, a formula of estimation is established for muscle mass of the upper limb where, MMES $(\mathrm{kg})=(($ Height - Fold triceps $) \times(0.043 \times$ perimeter relaxed $\mathrm{arm}^{2}$ ) - Weigh) / 1000, with a value $\mathrm{r}=0.83$ and one mistake estimated of $0,61 \mathrm{~kg}$. For the muscle mass for low limb it is established, MMEI (kg) = (Height - adds folds thigh and leg) $\mathrm{x}$ diameter femoral ${ }^{2}+($ Perimeter average thigh + Perimeter leg $) /$ 1000 , with $\mathrm{r}=0.74$ and estimation error of $1.2 \mathrm{~kg}$. The determination of these components by segment, improve the specificity of the anatomical knowledge of individuals of different populations, particularly in sportsmen, who defer his distribution of tissue according to the sports discipline and at a very low cost, since anthropometry does.

KEY WORDS: Anthropometry; Muscle mass; Dual Energy X ray Absorsiometry. 


\section{REFERENCIAS BIBLIOGRÁFICAS}

Aloia, J. F.; Vaswani, A.; Ma, R. \& Flaster, E. To what extent is bone mass determined by fat-free or fat mass? Am. J. Clinical Nutrition, 61:1110-4, 1995.

Brunton, J.; Bayley, H. \& Atkinson, S. Validation and application of dual-energy $\mathrm{x}$-ray absorptiometry to measure bone mass and body composition in small infants. Am. J. Clinical Nutrition, 58:839-45, 1993.

Drinkwater, D. T. An anatomically deroved method for the anthropometric estimation of human body composition. Ph.D. Thesis, Simon Fraser University, 1984.

Drinkwater, D. T.; Martin A. R.; Ross, W. D. \& Clarys, J. P. Validation by cadaver dissection of Matiegka's equations for the anthropometric estimation of anatomical body composition in human adults. In: Day, J. A. P. (Ed.) Perspectives in Kinanthropometry. Champaign, Human Kinetics, 1985. pp.221-7.

Haarbo, J.; Gotfredsen, A.; Hassager, C. \& Christiansen, C. Validation of body composition by dual energy X-ray absorptiometry (DEXA). Clin. Physiol., 11:331-41, 1991.

Heymsfield, S. B.; Smith, R.; Aulet, M.; Bensen, B.; Lichtman, S.; Wang, J.; et al. Appendicular skeletal muscle mass: measurement by dual-photon absorptiometry. Am. J. Clin. Nutr., 52:214-8, 1990.

Jensen, M. D.; Kanaley, J. A.; Roust, L. R.; O'Brien, P. C.; Braun, J. S.; Dunn, W. L.; et al. Assessment of body composition with use of DEXA: evaluation and comparison with other methods. Mayo Clin. Proc., 68:867-73, 1993.

Kerr, D. A. An anthropometric method for the fractionation of skin, adipose, muscle, bone and residual tissue masses in males and females age 6 to 77 years. M.Sc. Thesis, Simon Fraser University, 1988.

Kim, J.; Wang, Z.; Heymsfield, S. B.; Baumgartner, R. N. \& Gallagher, D. Total-body skeletal muscle mass: estimation by a new dual-energy x-ray absorptiometry method. Am. J. Clin. Nutr., 76:378-83, 2002.

Kim, J.; Heshka, S.; Gallagher, D.; Kotler, D.; Mayer, L.; Albu, J.; et al. Intermuscular adipose tissue-free skeletal muscle mass: estimation by dual-energy X-ray absorptiometry in adults. J. Appl. Physiol., 97:655-60, 2004.
Korth, W. Preliminary evidence that DEXA provides an accurate assessment of body composition. J. Appl. Physiol., 84:372-7, 1998.

Levine, J.; Abboud, L.; Barry, M.; Reed, J.; Sheedy, P. \& Jensen, M. Measuring leg muscle and fat mass in humans: comparison of CT and dual-energy X-ray absorptiometry. J. Appl. Physiol., 88:452-6, 2000.

Lohman, T. G. \& Chen, Z. Dual energy X-Ray Absorptiometry. In: Heymsfield, S. B.; Lohman, T. G.; Wang, Z. M. \& Going, S. B. (Eds.). Human Body Composition. Champaign, Human Kinetics, 2005. pp.6378.

Martin, A. P.; Spenst, L. F.; Drinkwater, D. T. \& Clarys, J. P. Anthropometric estimation of muscle mass in men. Med. Sci. Sports Exerc., 22:729-33, 1990.

Ross, W. D., Ward, R.; Leathy, R. M. \& Day, J. A. P. Proportionality fo Montreal Athletes. In: Carter, J. E. L. (Eds.). Physical structure of Olympic athletes, Part I: The Montreal Olympic Games anthropological project. Basel, Karger, 1982.

Shih, R.; Wang, Z.; Heo, M.; Wang, W. \& Heymsfield, S. B. Lower limb skeletal muscle mass: development of dualenergy X-ray absorptiometry prediction model. J. Appl. Physiol., 89:1380-6, 2000.

Visser, M.; Fuerst, T.; Lang, T.; Salamone, L. \& Harris, T. Validity of fan-beam dual-energy X-ray absorptiometry for measuring fat-free mass and leg muscle mass. J. Appl. Physiol., 87:1513-20, 1999.

Dirección para correspondencia:

Fernando Rodríguez Rodríguez

Avda. El Bosque 1290

Casilla 4059

Pontificia Universidad Católica de Valparaíso

Valparaiso

CHILE

Email: fernando.rodriguez@ucv.cl

Recibido : 10-09-2010

Aceptado: $11-10-2010$ 